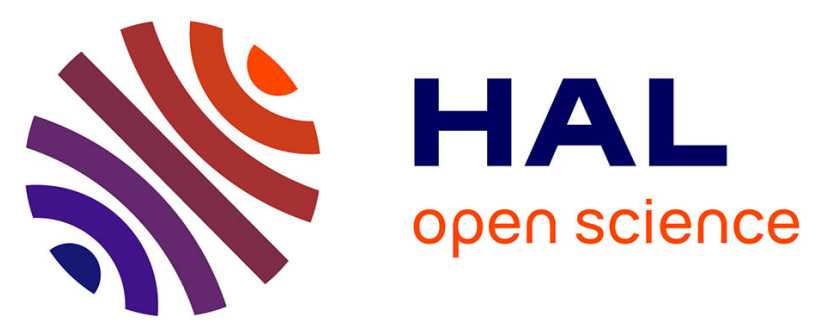

\title{
Deep sequencing shows that oocytes are not prone to accumulate mtDNA heteroplasmic mutations during ovarian ageing
}

L. Boucret, C. Bris, Valérie Seegers, D. Goudenège, V. Desquiret-Dumas, M. Domin-Bernhard, V Ferré-L'Hotellier, P. E Bouet, P. Descamps, P. Reynier, et al.

\section{To cite this version:}

L. Boucret, C. Bris, Valérie Seegers, D. Goudenège, V. Desquiret-Dumas, et al.. Deep sequencing shows that oocytes are not prone to accumulate mtDNA heteroplasmic mutations during ovarian ageing. Human Reproduction, 2017, 32 (10), pp.2101 - 2109. 10.1093/humrep/dex268 . inserm01815346

\section{HAL Id: inserm-01815346 https://www.hal.inserm.fr/inserm-01815346}

Submitted on 14 Jun 2018

HAL is a multi-disciplinary open access archive for the deposit and dissemination of scientific research documents, whether they are published or not. The documents may come from teaching and research institutions in France or abroad, or from public or private research centers.
L'archive ouverte pluridisciplinaire HAL, est destinée au dépôt et à la diffusion de documents scientifiques de niveau recherche, publiés ou non, émanant des établissements d'enseignement et de recherche français ou étrangers, des laboratoires publics ou privés. 


\section{Deep sequencing shows that oocytes are not prone to accumulate mtDNA heteroplasmic mutations during ovarian ageing}

\section{Boucret ${ }^{1,2}$, C. Bris ${ }^{2,3}$, V. Seegers ${ }^{4}$, D. Goudenège ${ }^{2,3}$, V. Desquiret- Dumas $^{2,3}$, M. Domin-Bernhard ${ }^{5}$, V. Ferré-L'Hotellier', P.E. Bouet ${ }^{6}$, P. Descamps ${ }^{6}$, P. Reynier ${ }^{2,3}$, V. Procaccio ${ }^{2,3}$, and P. May-Panloup ${ }^{1,2, *}$}

'Laboratoire de Biologie de la Reproduction, Centre Hospitalier Universitaire d'Angers, 49933 Angers cedex 9, France ${ }^{2}$ Institut MITOVASC, CNRS 6015, INSERM U 1083, Université d'Angers, 49933 Angers, France ${ }^{3}$ Département de Biochimie et Génétique, Centre Hospitalier Universitaire d'Angers, 49933 Angers cedex 9, France ${ }^{4}$ Département de Biométrie, Institut de Cancérologie de l'Ouest, CRCINA—Unité Inserm UI 232-Equipe 7, 49100 Angers, France ${ }^{5}$ Département de Gynécologie Obstétrique et Reproduction Humaine, CHU de Rennes, F-35033 Rennes, France ${ }^{6}$ Service de Gynécologie-Obstétrique, Centre Hospitalier Universitaire d’Angers, 49933 Angers cedex 9, France

*Correspondence address. Laboratoire de Biologie de la Reproduction, Centre Hospitalier Universitaire d'Angers, 49933 Angers cedex 9 , France. E-mail: pamaypanloup@chu-angers.fr

Submitted on March 30, 20 I 7; resubmitted on June 15, 201 7; accepted on July 28, 2017

STUDY QUESTION: Does ovarian ageing increase the number of heteroplasmic mitochondrial DNA (mtDNA) point mutations in oocytes?

SUMMARY ANSWER: Our results suggest that oocytes are not subject to the accumulation of mtDNA point mutations during ovarian ageing.

WHAT IS KNOWN ALREADY: Ageing is associated with the alteration of mtDNA integrity in various tissues. Primary oocytes, present in the ovary since embryonic life, may accumulate mtDNA mutations during the process of ovarian ageing.

STUDY DESIGN, SIZE, DURATION: This was an observational study of 53 immature oocyte-cumulus complexes retrieved from 35 women undergoing IVF at the University Hospital of Angers, France, from March 2013 to March 20I4. The women were classified in two groups, one including 19 women showing signs of ovarian ageing objectified by a diminished ovarian reserve (DOR), and the other, including 16 women with a normal ovarian reserve (NOR), which served as a control group.

PARTICIPANTS/MATERIALS, SETTING, METHODS: mtDNA was extracted from isolated oocytes, and from their corresponding cumulus cells (CCs) considered as a somatic cell compartment. The average mtDNA content of each sample was assessed by using a quantitative real-time PCR technique. Deep sequencing was performed using the lon Torrent Proton for Next-Generation Sequencing. Signal processing and base calling were done by the embedded pre-processing pipeline and the variants were analyzed using an in-house workflow. The distribution of the different variants between DOR and NOR patients, on one hand, and oocyte and CCs, on the other, was analyzed with the generalized mixed linear model to take into account the cluster of cells belonging to a given mother.

MAIN RESULTS AND THE ROLE OF CHANCE: There were no significant differences between the numbers of mtDNA variants between the DOR and the NOR patients, either in the oocytes $(P=0.867)$ or in the surrounding $C \mathrm{Cs}(P=0.154)$. There were also no differences in terms of variants with potential functional consequences. De-novo mtDNA variants were found in $28 \%$ of the oocytes and in $66 \%$ of the CCs with the mean number of variants being significantly different (respectively $0.32 \mathrm{I}, \mathrm{SD}=0.547$ and $\mathrm{I} .075, \mathrm{SD}=\mathrm{I} . \mathrm{I} 58)(P<0.000 \mathrm{I})$. Variants with a potential functional consequence were also overrepresented in CCs compared with oocytes $(P=0.0019)$.

LARGE SCALE DATA: N/A.

(C) The Author 2017. Published by Oxford University Press on behalf of the European Society of Human Reproduction and Embryology. All rights reserved.

For Permissions, please e-mail: journals.permissions@oup.com 
LIMITATIONS, REASONS FOR CAUTION: Limitations may be due to the use of immature oocytes discarded during the assisted reproductive technology procedure, the small size of the sample, and the high-throughput sequencing technology that might not have detected heteroplasmy levels lower than $2 \%$.

WIDER IMPLICATIONS OF THE FINDINGS: The alteration of mtDNA integrity in oocytes during ovarian ageing is a recurring question to which our pilot study suggests a reassuring answer.

STUDY FUNDING/COMPETING INTEREST(S): This work was supported by the University Hospital of Angers, the University of Angers, France, and the French national research centers, INSERM and the CNRS. There are nocompeting interests.

Key words: diminished ovarian reserve / mitochondria / mtDNA / oocyte / ovarian ageing

\section{Introduction}

Mitochondria constitute the powerhouse of cells, producing the energy required for all cellular functions. In addition to their role in producing ATP, these organelles play a central role in apoptosis, calcium regulation, and thermogenesis, as well as in cell signaling pathways and gene expression (Gut and Verdin, 2013). Each mammalian somatic cell contains several hundred mitochondria that form a continuously dividing and recombining network containing hundreds of thousands of mitochondrial DNA (mtDNA) copies.

In humans, mtDNA is a double-stranded, circular 16569 bp DNA molecule (Anderson et al., 198I), coding for 13 essential subunits of the respiratory chain complexes, as well as 22 tRNAs, and 2 rRNAs, constituting part of the mitochondrial translation machinery. MtDNA is transmitted according to a non-Mendelian maternal mode with a genome exclusively transmitted through oocytes (Giles et al., 1980).

The oocyte has the richest mtDNA content of all human cells, containing up to several hundred thousand copies (Otten and Smeets, 20I5). This mtDNA content is formed by the stochastic distribution of the few (only about one hundred) mtDNA molecules present in primordial germ cells during oogonial divisions, combined with an amplification of selected molecules during oogenesis and folliculogenesis (Wai et al., 2008; Mishra and Chan, 2014; Stewart and Chinnery, 20I5). This process has led to the 'bottleneck theory' (Hauswirth and Laipis, 1985) according to which a very small number of mtDNA molecules may populate the oocyte and consequently the entire organism. This theory explains how mtDNA may be 'refreshed' and 'purified' from one generation to another (Jansen and de Boer, 1998; Mishra and Chan, 2014; Stewart and Chinnery, 2015). Whereas this bottleneck has been extensively studied for the transmission of mtDNA mutations carried by the mother, its protective role against de-novo mutations of the oocyte is much less known. MtDNA is particularly susceptible to accumulate mutations, with the mutation rate in humans being almost 25 times higher than that of nuclear DNA (Lynch et al., 2006), mainly because of the proximity of mtDNA with the respiratory chain that produces reactive oxygen species (ROS) and the lack of protective histones and efficient repair mechanisms.

Ageing in mammals is correlated with a decline in the mitochondrial respiratory chain function and the accumulation of somatic mtDNA deletions and mutations (Krishnan et al., 2007; Trifunovic and Larsson, 2008). In this study, we were specifically interested in the potential effect of ovarian ageing on the integrity of mtDNA in oocytes compared to the somatic cell compartment, i.e. for each oocyte, its corresponding CCs. Interestingly, in the ovary, the mtDNA molecules in the oocyte and the surrounding CCs are subject, in parallel, to two successive events: first, a long stagnation in the 'resting phase', and second, a multiplication during the growth phase of the oocyte and during the multiplication phase of the CCs during folliculogenesis.

Ovarian ageing is characterized by a diminished ovarian reserve (DOR), which combines the quantitative and qualitative alterations of the ovarian oocyte pool (te Velde and Pearson, 2002). Ovarian ageing occurs more or less early in women depending on the influence of environmental and genetic factors not yet well defined (te Velde and Pearson, 2002; Broekmans et al., 2009). The great variability between women led us to consider the patient's ovarian reserve rather than merely the chronological age to evaluate the effect of ovarian ageing on mtDNA integrity.

We used the deep sequencing technique to evaluate heteroplasmic point mutations in 53 isolated oocytes and their corresponding CCs, obtained from two groups of women, one with a DOR and the other with a normal ovarian reserve (NOR).

\section{Materials and Methods}

\section{Characteristics of the patients}

Oocytes and CCs were obtained from 35 patients undergoing IVF with ICSI at the ART Center of the University Hospital of Angers, France, between March 2013 and March 2014. The Center proposes ICSI in cases of infertility, which represent about $50 \%$ of our IVF activity.

Among the 35 patients included, 19 presented a DOR whereas 16 had a NOR. The inclusion criteria used for classifying the patients in the DOR or NOR groups included the levels of the anti-Müllerian hormone (AMH), the $\mathrm{FSH}$, and estradiol, together with the ultrasound measurement of the ovarian antral follicle count (AFC) (de Carvalho et al., 2008). Patients were assigned to the NOR group if their AMH levels were above $2 \mathrm{ng} / \mathrm{ml}$, FSH levels below $8 \mathrm{IU} / \mathrm{l}$, estradiol levels below $60 \mathrm{pg} / \mathrm{ml}$ and the AFC > 4 per ovary. Patients were assigned to the DOR group if their AMH levels were below $2 \mathrm{ng} / \mathrm{ml}$ or if the AFC was $<5$ per ovary. All the women included in the study had both ovaries intact, with no history of ovarian surgery, autoimmune disease or gonadotoxic treatment that might have contributed to a DOR. Table I summarizes the characteristics of the 35 patients, as well as the procedures used for the suppression of pituitary gonadotrophin release [using $\mathrm{GnRH}$ antagonists, i.e. Cetrorelix (Cetrotide ${ }^{\circledR}$; Merck-Serono, Geneva, Switzerland) or Ganirelix (Orgalutran ${ }^{\circledR}$; Organon, Oss, Netherlands) or GnRH agonists, i.e. Triptoreline (Decapepty| ${ }^{\circledR}$; Ipsen Pharma, Paris, France)] and follicular growth stimulation [using FSH $+\mathrm{LH}$, i.e. Menotropine (Menopur ${ }^{\circledR}$; Ferring Pharmaceuticals, Copenhagen, Denmark) or Follitropine alpha + Lutropine alpha (Pergoveris ${ }^{\circledR}$; Merck-Serono, Geneva, Switzerland) or rFSH, i.e. Follitropine alpha (Gonal- $f^{\circledR}$;Merck-Serono, Geneva, Switzerland) or 
Table I Characteristics of the 35 patients studied.

\begin{tabular}{|c|c|c|c|c|}
\hline & \multirow{2}{*}{$\begin{array}{l}\text { Overall } \\
\ldots=35\end{array}$} & \multirow{2}{*}{$\begin{array}{l}\text { NOR } \\
N=16\end{array}$} & \multirow{2}{*}{$\begin{array}{l}\text { DOR } \\
N=19\end{array}$} & \multirow[t]{2}{*}{$P$-value } \\
\hline & & & & \\
\hline Age, median (IQR) & $34(29.5-36)$ & $31(29-32.5)$ & $36(32.5-37)$ & 0.0144 \\
\hline Age range & $25-42$ & $28-38$ & $25-42$ & \\
\hline Mean (SD) & $33.1(3.9)$ & $31.4(2.8)$ & $34.5(4.2)$ & \\
\hline BMI, median (IQR) & $20.8(20.2-23.1)$ & $21.2(20.6-22.9)$ & $20.6(19.6-23.1)$ & 0.4658 \\
\hline \multicolumn{5}{|l|}{ Tobacco } \\
\hline Non-smokers & $24(72.7 \%)$ & $10(30.3 \%)$ & $14(42.4 \%)$ & 0.4636 \\
\hline Smokers & $5(15.2 \%)$ & $2(6.1 \%)$ & $3(9.1 \%)$ & \\
\hline Former smokers & $4(12.1 \%)$ & $3(9.1 \%)$ & I (3.0\%) & \\
\hline Information missing & & I & I & \\
\hline E2 (pg/ml), median (IQR) & $31.5(23.1-47.8)$ & $33(25-57)$ & $30(20.5-46)$ & 0.5706 \\
\hline FSH (UI/I), median (IQR) & $8(6.4-9.5)$ & $7.0(5.2-7.4)$ & $8.8(8 .|-| \mid .2)$ & 0.0008 \\
\hline LH (UI/I), median (IQR) & $4.9(3.9-6.6)$ & $4.7(4.2-5.3)$ & $5.7(3.9-6.6)$ & 0.3002 \\
\hline AMH (ng/ml), median (IQR) & I.7। (I.4-2.35) & $2.9(2.16-3.9)$ & $1.4(0.98-1.53)$ & $<0.000$ I \\
\hline AFC, median (IQR) & II (8.5-2I) & $22(19-24.5)$ & $9(7-10)$ & $<0.0001$ \\
\hline Initial dose of FSH, median (IQR) & $300(200-300)$ & $200(150-225)$ & $300(300-325)$ & $<0.000$ I \\
\hline Total dose of FSH administered (UI), median (IQR) & $2512(1800-3200)$ & I744 (|575-208I) & $3150(2850-3338)$ & $<0.000$ I \\
\hline Number of OCCs retrieved & $8(5-10)$ & $10.5(8.8-13)$ & $5(4-7.5)$ & 0.0003 \\
\hline$\%$ of mature oocytes, median (IQR) & $77(60-82)$ & $80(76.5-88.5)$ & $67(50-80)$ & 0.0086 \\
\hline \multicolumn{5}{|l|}{ LH suppression } \\
\hline Agonist & $9(25.7 \%)$ & $6(37.5 \%)$ & $3(15.8 \%)$ & 0.282 \\
\hline Antagonist & $26(74.3 \%)$ & $10(62.5 \%)$ & $16(84.2 \%)$ & \\
\hline \multicolumn{5}{|l|}{ Stimulation } \\
\hline FSH & $25(71.4 \%)$ & $12(75 \%)$ & $13(68.4 \%)$ & 0.9572 \\
\hline $\mathrm{FSH}+\mathrm{LH}$ & $10(28.6 \%)$ & $4(25 \%)$ & $6(31.6 \%)$ & \\
\hline
\end{tabular}

IQR = interquartile range: 25 th-75th percentiles.

The differences in bold were considered significant at $P<0.05$.

Follitropine beta (Puregon ${ }^{\circledR}$; Organon, Oss, Netherlands)]. In each case, ovulation was induced with Ovitrelle ${ }^{\circledR}$ (Choriogonadotropine alfa; MerckSerono, Geneva, Switzerland), and the oocytes were retrieved transvaginally under ultrasound guidance.

\section{Isolation of oocytes and cumulus cells}

A total of 53 oocyte-cumulus complexes (OCCs) were collected, 27 among the NOR group, 26 among the DOR group. For each of the OCCs retained, we collected the oocyte, on one hand, and the corresponding CCs, on the other, for individual studies. Among the 53 OCCs, 3 were obtained from each of 4 women, 2 from each of 10 women and I from each of 21 women. The OCCs were retrieved $36 \mathrm{~h}$ after treatment with human chorionic gonadotrophin treatment, and washed in multiple dishes with Flushing medium (Origio-France, Limonest, France) to eliminate the remaining mural granulosa cells, blood cells, and cellular debris. The OCCs were subsequently incubated for $\mathrm{I} h$ in Ferticult culture media (Fertipro, Beernem, Belgium). For each patient, the oocytes were individually stripped using hyaluronidase ( $80 \mathrm{IU}$; Fertipro) and gentle pipetting with a I $25 \mu \mathrm{m}$ diameter stripper pipette (Origio, France). Metaphase II oocytes were used for the ICSI procedure. Oocytes which had not extruded their first polar body $2 \mathrm{~h}$ after removal of the CCs, i.e. germinal vesicles blocked at prophase I (GVs) or oocytes blocked after prophase I (GVBDs), were retained for our study. There were 25 oocytes in prophase I, and 28 were blocked between prophase I and metaphase II. Each oocyte was placed in $5 \mu \mathrm{l}$ Ferticult culture media and immediately frozen in liquid nitrogen until extraction of the nucleic acid. CCs were recovered in $600 \mu \mathrm{l}$ of Ferticult culture media and centrifuged at $2000 \mathrm{~g}$ for $10 \mathrm{~min}$. The supernatant was removed and the CC pellets were immediately frozen in liquid nitrogen until extraction of the nucleic acid.

\section{Extraction of DNA}

Total DNA was extracted from isolated oocytes and the corresponding CC fractions using the Nucleospin ${ }^{\circledR}$ tissue Kit (Macherey-Nagel ${ }^{\circledR}$, Hoerdt, France) according to the manufacturer's recommendations. Genomic DNA was finally eluted in $60 \mu$ l of elution buffer.

\section{Quantification of mtDNA copy numbers}

To determine the mtDNA copy number, real time quantitative PCR (QPCR) was performed using the Chromo4 System (Biorad ${ }^{\circledR}$, Hercules, CA, USA) in a $20 \mu$ reaction volume containing $\times$ I IQ SYBR Green Supermix $\left(\right.$ Biorad $\left.^{\circledR}\right)$ and a final concentration of $0.5 \mu \mathrm{M}$ of each gene-specific primer and $3 \mu$ of template. The pairs of primers selected were, respectively, FIRI (forward I and reverse I; nucleotide positions 8342-8359 and 8546-8559) to quantify the mtDNA in oocytes and in CCs, and NCOA3 F/R (nuclear receptor coactivator three forward: 5'-AAAGTTAATGGAGTTTCCTG-3' and reverse: 5'-AGGACTGGCGTTTATGTCTT-3') to quantify the 
nuclear DNA in CCs. The reactions were performed as described in Supplementary Table SI.

The presence of the common $4977 \mathrm{bp} m \mathrm{mDNA}$ deletion (from nucleotide 8469 to nucleotide 13447 ) was excluded in the tested samples (Poe et al., 2007). Briefly, the non-deleted mtDNA was amplified by the FIRI method, and the deleted mtDNA was amplified with the FIR2 method (forward I and reverse 2; nucleotide positions 8342-8359 and I5 727-I5 749 ) by quantitative PCR. The common deletion was considered absent for a ratio of $<0.1 \%$ between the $m t D N A$ copy numbers found with the FIRI and FIR2 methods.

\section{Ion proton sequencing}

\section{Compilation of the mtDNA library}

The whole mitochondrial genome was amplified in two overlapping fragments of 8009 bp (amplicon A) and 8994 bp (amplicon B). The primer pairs used for both PCRs, tested on DNA extracted from cells devoid of mtDNA-(Rho Zero cells) to avoid pseudogene amplification, were located on the cytochrome $b$ and cytochrome $c$ oxidase one genes: 5'-TACGTTG TAGCCCACTTCCACT-3' and 5'-GCCCGATGTGTAGGAAGAG-3' for amplicon A; 5'-AACTTCGGCTCACTCCTTGG-3' and 5'-AGTAAC GTCGGGGCATTCCG-3' for amplicon B. Long-range PCR was performed with KapaLongRange DNA polymerase according to the manufacturer's recommendations (Kapa Biosystems, Boston, MA, USA). Agarose gel electrophoresis (I\%) was used to confirm the presence of PCR products. The concentrations of PCR products were quantified by the Qubit ${ }^{\circledR}$ 2.0 Fluorometer using the Qubit ${ }^{\circledR}$ dsDNA HS Assay Kit (Thermo Fischer technologies, Waltham, MA, USA). For each sample, amplicons $A$ and $B$ were pooled in an equimolar manner. Libraries were compiled from 50 to $200 \mathrm{ng}$ of pooled PCR products with the enzymatic fragmentation method of the Library Builder Automate using the dedicated Ion Xpress ${ }^{\mathrm{TM}}$ Plus Fragment Library Kit (Thermo Fischer technologies). All purification procedures were performed using Agencourt AMpure XP beads (Beckman Coulter, Brea, CA, USA).

\section{Emulsion PCR, enrichment and sequencing}

Emulsion PCR and enrichment were performed on pooled libraries diluted to $70-100$ ppM using the lon Chef Automate (Thermo Fischer technologies). The enriched mtDNA samples were sequenced using an ion proton high-throughput sequencing platform according to the manufacturer's protocol (Thermo Fischer technologies). The possibility of contamination was excluded by the use of negative controls with water instead of the amplified mtDNA.

\section{Processing the sequencing data}

Sequencing was performed using lon Torrent Proton, and the signal processing and base calling was done by the pre-processing embedded pipeline. Demultiplexed reads were mapped on an mtDNA reference sequence (NC_012920) before being analysed with a dedicated in-house pipeline integrating various modules for coverage analysis, variant calling, annotation and prioritization. The calling module uses a consensus-based approach and integrates the prediction of six callers (Torrent Suite Variant Caller, GATK [PMID:20644I99], VarScan [PMID:25553206], SNVer [PMID:2I8I3454], LoFreq [PMID:23066I08] and Platypus [PMID:25017I05]). All of the variant calling formats (VCFs) generated were normalized and decomposed before launching the annotation-prioritization module which combines the NCBI Variant Reporter for gene annotation and ANNOVAR [PMID:250I7I05] for variant prioritization. ANNOVAR allowed the inclusion of several databases, i.e. Mitomap [PMID:25489354] and Mitlmpact2 [PMID:255I6408] and prioritization tools, i.e. Polyphen2 [PMID:233I5928], SIFT [PMID:1956I590] and MutationTaster [PMID:2468I72I]. All the results were then included in a private database that may be interrogated via a dedicated graphical user interface for reducing the number of candidate variants by applying prioritization filters. Putative variants were further filtered using the following criteria: at least three callers predicting the variant, including the Torrent Suite Variant Caller, coverage greater than $\times 500$, quality score $>I 50$ and a minimum mtDNA heteroplasmy level of $2 \%$, was validated as a sensitive threshold for the detection of variants. Only substitution variants were studied.

\section{Statistical analysis}

For the characteristics of patients, categorical variables were described using percentages and were compared using Chi-squared tests or Fisher's exact tests as appropriate. Quantitative and discrete variables were described using the median and the interquartile range (IQR), i.e. values between the 25th and 75th percentiles, and were compared using the Mann-Whitney test. The association between age and the number of variants was assessed using a linear regression model.

To compare the characteristics of the oocytes and the surrounding CCs, we also computed a mixed generalized linear regression model (GLMM) to take into account the cluster of oocytes and CCs belonging to a given mother (with a random effect), and recorded the $P$-values corresponding to the factors studied. All the statistical tests were bilateral and the differences were considered significant at $P<0.05$. All analyses were computed using R version 3.3.2 (R Core Team, 2016) and the Ime4 packages (Bates et al., 20I5).

\section{Ethical approval}

All participants gave their written informed consent, and the collection of OCCs was authorized by the Ethical Committee of the University Hospital of Angers, France (Number DC-20I4-2224).

\section{Results}

\section{Patients}

As expected, the AMH and AFC levels were significantly lower and the FSH levels significantly higher in the DOR group than in the NOR group of patients since these were precisely the criteria used for classifying the patients into the two groups. Not surprisingly, the doses of FSH used for the stimulation phase were higher in the DOR group than in the NOR group of patients. However, there was no significant difference with respect to the drugs used for suppressing $\mathrm{LH}$ and to the ovarian stimulation between the two groups of patients (Table I).

The age of the patients was significantly lower in the NOR group (median 3I years; IQR: 29-32.5 years) than in the DOR group (median 36 years; IQR: 32.5-37 years). However, there were no significant differences between the two groups in terms of the BMI or addiction to tobacco smoking, both of which are likely to adversely affect ovarian reserve (Table I).

The mean number of OCCs retrieved was lower in the DOR group than in the NOR group, and the percentage of immature oocytes was significantly different between the two groups of patients (Table I). However, the numbers of GVs and GVBDs studied was similar in the DOR and the NOR groups (Table II).

\section{Analysis of OCCs}

The overall mean number of OCCs (oocytes and corresponding CCs) studied per patient was I.5I (SD =0.7), with no significant difference between the NOR and DOR patients for which the mean numbers were respectively I.69 $(\mathrm{SD}=0.87)$ and I.37 $(\mathrm{SD}=0.5), P=0.36$. 
Table II MtDNA copy numbers in the oocytes and CCs of NOR and DOR patients.

\begin{tabular}{|c|c|c|c|c|}
\hline & Overall & NOR & DOR & $P$ value \\
\hline & $N=53$ & $N=27$ & $N=26$ & \\
\hline Number of GV/GVBD studied & $25 / 28$ & $14 / 13$ & $11 / 15$ & 0.6740 \\
\hline mtDNA in oocytes median (IQR) & II 4 I00 (85 820-138 200) & 125200 (93 320-137 100) & $93650(77360-139000)$ & 0.233 \\
\hline mtDNA per CC median (IQR) & $161(135-194)$ & $169(142.5-199)$ & $146(122.8-173.5)$ & 0.0953 \\
\hline mtDNA in the totality of CCs studied median (IQR) & $135200(68$ | $80-172300)$ & $135200(75040-186600)$ & $134300(67500-165400)$ & 0.588 \\
\hline
\end{tabular}

P-value from Mann-Whitney test for mtDNA.

The differences in bold were considered significant at $P<0.05$.

\section{The mtDNA common deletion was not present in our cohort of patients}

No 4977 bp common deletion was found either in the oocytes or in the CCs of the patients studied.

\section{The mtDNA copy numbers were not significantly different in the groups studied}

The mtDNA copy numbers per cell, in the oocytes and the CCs, were not significantly different in the DOR and the NOR groups (Table II).

The mtDNA copy numbers per cell, in the oocytes and the CCs, were not significantly different between GVs and GVBDs (Supplementary Table SII).

The mtDNA copy numbers on which our analyses of variants were performed were similar between the oocytes and the totality of the corresponding CCs studied (respectively, median: II4 I00; IQR: 85 820-138 200 and median: I35 200; IQR: 68 I80-172300, P=0.7I6) and between the DOR and the NOR groups in the totality of the CCs studied (Table II).

\section{MtDNA variants}

Variants identified both in the oocytes and in the corresponding CCs Eight variants were found in six oocytes and in the corresponding CCs (Fig. I and Supplementary Table SIII), with average proportions of heteroplasmy of $38.7 \%$ ( $\sigma=29.9 \%$, range: $2-94 \%)$ for the oocytes and $36.9 \%(\sigma=29.8 \%$, range: $2-91 \%)$ for the CCs.

Variants specifically identified in one of the two cellular types (specific variants)

There were 17 variants found in the oocytes only (Fig. I and Supplementary Table III), with an average proportion of heteroplasmy of $10.3 \%$ ( $\sigma=6.6 \%$, range: $3.8-20.6 \%$ ); these variants were found in 15 of the 53 oocytes studied (28\%) (Fig. 2).

There were 57 variants found in the CCs only (Fig. I and Supplementary Table III), with an average proportion of heteroplasmy of $9.6 \%$ ( $\sigma=8.7 \%$, range: $3.1-45.5 \%)$; these variants were found in 35 of the 53 CCs studied (66\%) (Fig. 2).

There was a significant difference between the mean number of variants in the oocytes and in the corresponding surrounding CCs in all of the patients and in each of the DOR and NOR groups of patients (Table III).
There was no significant difference between the mean number of specific variants in the DOR and NOR patients either in the oocytes or in the CCs (Table III).

There was also no relationship between chronological age and the number of variants in the oocyte $(P=0.320)$ or in CCs $(P=0.570)$.

No difference was found in the proportion of oocytes or CCs bearing variants according to the immaturity state of the oocyte (GV or GVBD): for oocytes, 5/I5 GV and I0/28 VGBD $(P=0.2049)$ and for CCs, 19/25 VG and 16/28 GVBD $(P=0.1479)$.

\section{Different types of specific variants}

We distinguished between the two main categories of variants without potential functional consequences, i.e. variants with non-coding regions or synonymous variants, and those with potential functional consequences affecting mRNAs, tRNAs and rRNAs in the oocytes and in the surrounding CCs (summarized in Fig. 2 and Supplementary Table SIII).

The proportion of variants with potential functional consequences was lower in the oocytes than in the corresponding surrounding CCs in all of the patients and in each group of the DOR and NOR patients (Table IV).

There was no significant difference between the proportions of variants with potential functional consequences in the DOR and NOR groups of patients, either in the oocytes or in the surrounding CCs (Table IV).

\section{Pathogenic mutations}

Among the variants identified, two pathogenic mutations have been definitely associated with mitochondrial diseases in the literature (for details, see Mitomap database http://www.mitomap.org). One is the m. I4459G > A mutation in the ND6 gene associated with the Leigh syndrome and Leber's Hereditary Optic Neuropathy (LHON) (3\% heteroplasmy), and the other is the m.586G $>$ A mutation in a tRNA (phe) gene responsible for a neurodegenerative disorder identified in two women with, respectively, 4 and 12\% heteroplasmy (Supplementary Table III). These three variants were seen only in the CCs, and in only one CC when several OCCs from the same patient were studied.

\section{Discussion}

Ovarian ageing may affect oocyte competence by targeting cytoplasmic components, including mitochondria, thereby entailing serious cellular consequences including nuclear chromosomal abnormalities (Meldrum et al., 2016). The effects of ovarian ageing on mitochondria in oocytes and in the corresponding CCs have attracted much attention in recent years and one of the most recurrent findings is the 

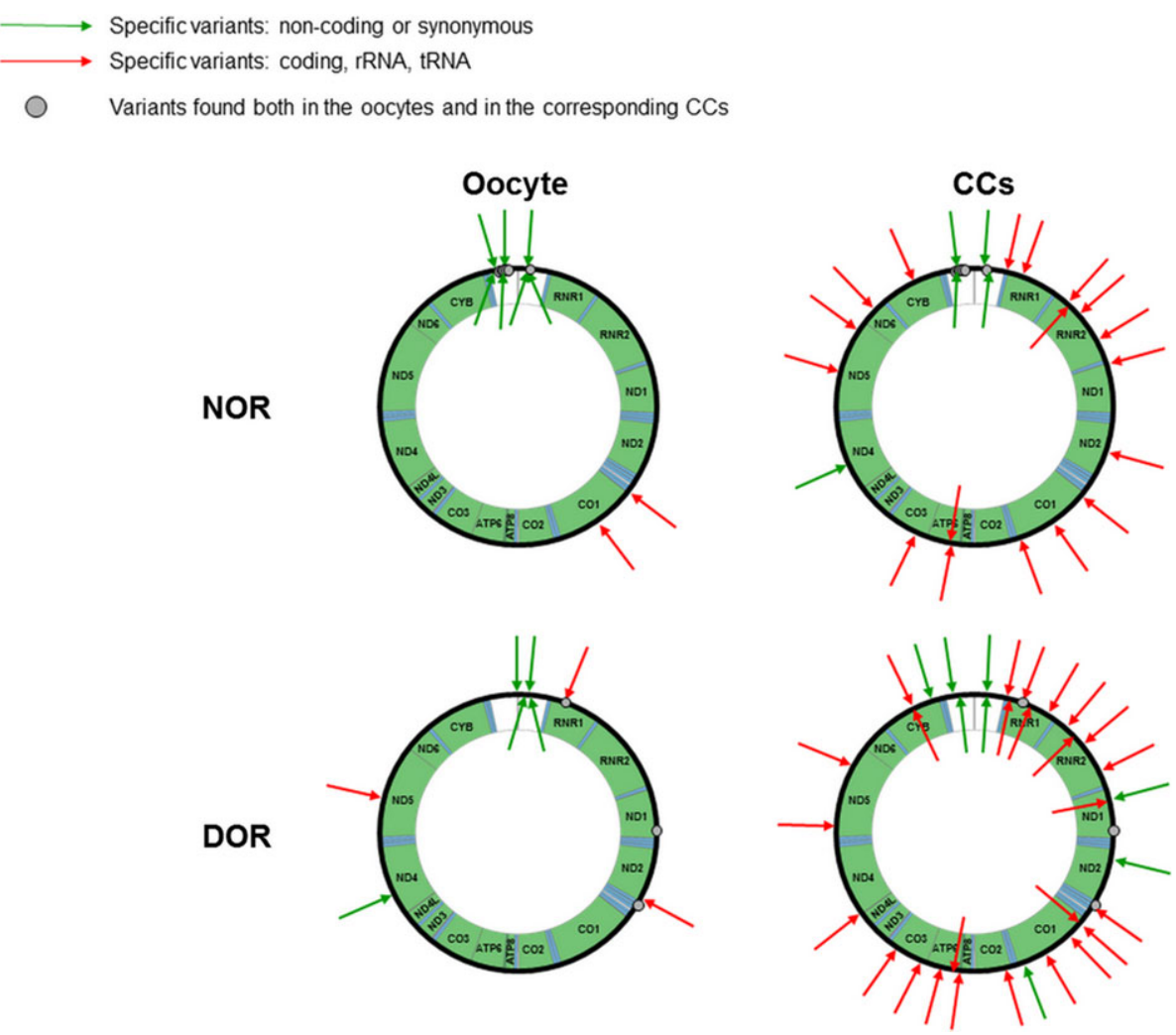

Figure I Representation of mtDNA, mitochondrial genes and variants found in oocytes or CCs. The gray dots symbolize the variants found in both the oocytes and in the corresponding CCs. The arrows symbolize the variants specifically found in one or the other cell types (green: non-coding variants or synonyms; red: coding tRNA or rRNA). RNR: rRNA; ND = NADH dehydrogenase, complex I; CYB = cytochrome b, complex III; CO = cytochrome oxidase, complex IV; and ATP = ATPase, complex V. The blue lines symbolize the 22 tRNAs.

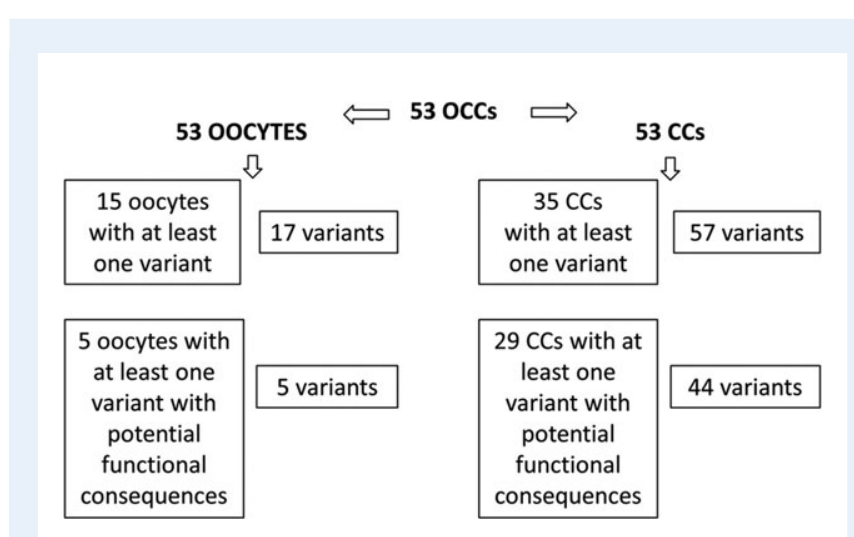

Figure 2 Flow chart showing the numbers of specific mtDNA variants in oocytes and CCs.

decrease in mtDNA copy numbers in oocytes with ovarian ageing (see review by May-Panloup et al., 2016).

Concerning the qualitative aspects, the link between oocyte mtDNA integrity and ovarian ageing remains controversial. Studies on oocytes from older women, have reported a greater number of mtDNA abnormalities, such as the accumulation of the common 4977-bp mtDNA deletion (Keefe et al., 1995; Chan et al., 2005), or some point mutations (Barritt et al., 2000), whereas other studies have found no difference in the number of deletions (Brenner et al., 1998; Barritt et al., 1999; Chan et al., 2005) or that of mutations (Fragouli et al., 20I5). However, in these studies, the mutational burden was very low and did not appear to be responsible for any direct functional outcome (Brenner et al., 1998). In effect, a pathogenic mtDNA mutation manifests itself only when the mutant load exceeds a certain threshold, leading to the appearance of symptoms. Nevertheless, the effects of low levels of heteroplasmy in the oocytes of older mothers may appear in the offspring (Rebolledo-Jaramillo et al., 2014). Thus, maternally inherited mtDNA mutations, associated with the combination of acquired somatic mutations, have been shown to be responsible for age-related disorders and defective brain development in mice (Ross et al., 20l3). Thus, it would be important to study oocyte heteroplasmy and its variation with ageing, reflected here by pool size.

Next-generation sequencing offers the great sensitivity required for the detection of low levels of heteroplasmy. To our knowledge, this is the first study carried out on humans using this technique to compare the variants present in oocytes to those in CCs. The sequencing was performed on an mtDNA copy number which was similar between the different groups compared, thus rendering the quantification of variants fully comparable. 
Table III Specific variants in oocytes and CCs, in DOR and NOR patients.

\begin{tabular}{|c|c|c|c|c|}
\hline & Oocytes and CCs & Oocyte & CCs & $P$ value GLMM model \\
\hline Overall mean number (SD) of specific variants in NOR and DOR patients & $0.698(0.9776)$ & $0.321(0.547)$ & $1.075(1.158)$ & $<0.0001$ \\
\hline Mean number (SD) of specific variants in NOR patients & $0.593(0.714)$ & $0.333(0.480)$ & $0.852(0.8 \mid 8)$ & 0.0064 \\
\hline Mean number (SD) of specific variants in DOR patients & $0.808(1.189)$ & $0.308(0.618)$ & $1.308(\mathrm{I} .408)$ & 0.0013 \\
\hline$P$-value GLMM model & 0.259 & 0.867 & 0.154 & \\
\hline
\end{tabular}

The GLMM regression takes into account clusters of oocytes or CCs belonging to a given mother.

The differences in bold were considered significant at $P<0.05$.

Table IV Specific variants with potential functional consequences (PFCs) in oocytes and CCs, in DOR and NOR patients.

\begin{tabular}{|c|c|c|c|c|}
\hline Variants with PFCs & $\begin{array}{l}\text { Oocytes and CCs with } \\
\text { specific variant }(N=50 *)\end{array}$ & $\begin{array}{l}\text { Oocyte }(N=15 \text { oocytes } \\
\text { with specific variants })\end{array}$ & $\begin{array}{l}\text { CCs }(N=35 \text { CCs with } \\
\text { specific variants })\end{array}$ & $\begin{array}{l}\text { P-value } \\
\text { GLMM model }\end{array}$ \\
\hline $\begin{array}{l}\text { In NOR and DOR patients } \\
\left(N=50^{*} \text { OCCs }\right)\end{array}$ & $34 / 50(68.0 \%)$ & $5 / 15(33.3 \%)$ & $29 / 35$ (85.3\%) & 0.0019 \\
\hline $\begin{array}{l}\text { In NOR patients ( } N=26 \\
\text { OCCs with specific variants) }\end{array}$ & I5/26 (57.7\%) & $2 / 9(22.2 \%)$ & $13 / 17(76.5 \%)$ & 0.0135 \\
\hline $\begin{array}{l}\text { In DOR patients ( } N=24 \\
\text { OCCs with specific variants) }\end{array}$ & 19/24 (79.2\%) & $3 / 6(50 \%)$ & 16/18 (88.9\%) & 0.0785 \\
\hline$P$-value GLMM model & 0.1859 & 0.3287 & 0.4018 & \\
\hline
\end{tabular}

The GLMM regression takes into account clusters of oocytes or CCs belonging to a given mother.

*Three OCCs were without any specific variants.

The differences in bold were considered significant at $P<0.05$.

\section{Heteroplasmic mtDNA variants found both in oocytes and corresponding CCs}

The eight variants in both oocytes and corresponding CCs (Fig. I and Supplementary Table SIII) potentially correspond to the variants preexisting in the mother, which could be transmitted via the oocyte to the offspring without elimination through the mitochondrial bottleneck. When several OCCs obtained from a given woman were studied, these eight variants were found in all of the oocytes and the CCs, except for one variant that was found in only one of the three OCCs. However, the level of heteroplasmy of this variant was only $3 \%$ in the oocyte, and it is possible that the variant escaped detection in the other OCCs because of the threshold of sensitivity of the technique used.

In one further case, the same variant was retrieved in several CCs but not found in the corresponding oocytes. It is interesting to note that this variant corresponded to a non-synonymous mutation (m.6027G > A; G42S) whereas the other variants were five noncoding variants, one synonymous variant that did not result in an amino acid change, and two variants involving either a tRNA gene or a rRNA gene. Indeed, it has been shown that the purifying selection that occurs during the bottleneck process preferentially targets proteincoding genes rather than tRNA genes (Stewart and Larsson, 20I4).

\section{Heteroplasmic mtDNA variants found only either in oocytes or corresponding CCs}

Among the 53 oocytes studied, 15 contained a total of 17 variants, i.e. $28 \%$ of the oocytes carried at least one mtDNA variant (Fig. 2). These variants (Fig. I and Supplementary Table SIII), found only in the oocytes, are unlikely to have been inherited from the mother, since they were found neither in the corresponding CCs nor in the other oocytes or CCs from the same women. More probably, they correspond to mutations acquired during germ line quiescence or development. This result is in accordance with previous studies showing mtDNA mutations in $27 \%$ of human oocytes (Jacobs et al., 2007), and de novo variants in $20 \%$ of zebra fish oocytes (Otten et al., 2016).

In contrast, mtDNA variants were significantly more frequent in CCs with 57 variants found in 35 of the 53 OCCs studied, i.e. $66 \%$ of CCs carried at least one variant (Fig. 2). These CC variants (Fig. I and Supplementary Table SIII) were either mutations that were counterselected in the corresponding oocytes by the bottleneck mechanism or acquired during folliculogenesis. The former hypothesis appears less likely since all these variants, with the exception of the one discussed above, i.e. m.6027G > A; G42S, were found in only one CC when several OCCs from the same patient were studied.

Despite the similarity of the kinetics of mtDNA replication and the environmental conditions of the oocytes and the surrounding CCs, twice as many CCs as oocytes carried at least one mtDNA variant, and the mean number of variants was significantly higher in CCs than in oocytes $(P<0.000 \mathrm{I})$ (Table III). Interestingly, the percentage of variants potentially affecting gene expression, i.e. the non-synonymous, tRNA and rRNA variants was significantly higher in the CCs than in the oocytes $(P=0.0019)$ (Table IV). Moreover, the two pathogenic mutations associated with mitochondrial diseases, identified in our study, were found only in the CCs. Overall, oocytes seem spared from mtDNA damage in comparison to CCs. This could reflect either the 
protective role of CCs against oxidative stress in oocytes (Shaeib et al., 2016) or the action of the bottleneck effect in eliminating acquired variants. Interestingly, this phenomenon has been shown to continue during the growth phase of the oocyte, i.e. after the quiescent phase and up to a few months before ovulation (Wai et al. 2008).

There were no significant differences between the numbers of mtDNA variants between the DOR and the NOR patients, either in the oocytes or in the surrounding CCs (Table III). There were also no differences between the DOR and NOR patients in terms of variants with potential functional consequences (Table IV). Moreover, the number of variants was not correlated with the chronological age of the patients. Our findings concord with those of a recent study on a model of cloned cows, 3 and 10 years old, which did not find any significant accumulation of heteroplasmic mtDNA point mutations associated with ageing, although it did show an age-related increase in the number of mtDNA deletions in oocytes (Hammond et al., 2016). In our study, it could have been expected that the number of mutations in the DOR group may have been underestimated because of an increase in the $4977 \mathrm{pb}$ deletion both in oocytes and in the surrounding CCs. Indeed, this age-related deletion may eliminate a portion of the mitochondrial genome potentially carrying mutations (Tsai et al., 20I0). However, we did not identify the common deletion in our series of DOR patients.

This above results suggest that oocytes are not prone to accumulating $m t D N A$ heteroplasmic mutations during ovarian ageing.

In this study, we only used immature oocytes (because of ethical considerations) and cannot confirm that the over-representation of $m t D N A$ variants in CCs compared to oocytes also exists in mature oocytes. Indeed, due to the importance of mitochondrial functions in the cumulus with respect to the terminal maturation of the oocyte (Dalton et al., 2014), the mitochondrial dysfunction and mtDNA mutations in the CCs could be directly related to the lack of oocyte maturity. In contrast, in immature oocytes the absence of accumulation of mutations with ovarian aging is likely to be also found in mature oocytes. Moreover, our study may be also limited by the relatively small number of OCCs studied, and finally, because the highthroughput sequencing technology used may have failed to measure levels of heteroplasmy lower than $2 \%$ with sufficient reliability.

Nevertheless, our pilot study argues against the notion of the accumulation of mtDNA mutations in oocytes with ovarian ageing, although it remains a recurring and highly debated topic.

\section{Supplementary data}

Supplementary data are available at Human Reproduction online.

\section{Acknowledgements}

We are grateful to Kanaya Malkani for his critical reading and comments on the article.

\section{Authors' roles}

L.B., P.R., V.P. and P.M.P., the principal investigators, take primary responsibility for the article. L.B., C.B., P.R., V.P. and P.M.P. contributed to the conception, design and coordination of the research. P.E.B. and P.D. recruited the patients. L.B., V.S., D.G., V.D., M.D. and V.F.L. contributed to the collection and analysis of data. L.B., V.S., D.G.,
V.D., P.R., V.P. and P.M.P. contributed to writing the article. L.B., C.B., V.D., P.R., V.P. and P.M.P. contributed to the revision of the article, and the final version was approved by all the authors.

\section{Funding}

University Hospital of Angers, the University of Angers, France, and the French national research centers, Institut National de la Santé et de la Recherche Médicale (INSERM) and the Centre National de la Recherche Scientifique (CNRS).

\section{Conflict of interest}

None declared.

\section{References}

Anderson S, Bankier AT, Barrell BG, de Bruijn MH, Coulson AR, Drouin J, Eperon IC, Nierlich DP, Roe BA, Sanger F et al. Sequence and organization of the human mitochondrial genome. Nature 198I;290:457-465.

Barritt JA, Brenner CA, Cohen J, Matt DW. Mitochondrial DNA rearrangements in human oocytes and embryos. Mol Hum Reprod 1999;5:927-933.

Barritt JA, Cohen J, Brenner CA. Mitochondrial DNA point mutation in human oocytes is associated with maternal age. Reprod Biomed Online 2000; 1:96-100.

Bates D, Mächler M, Bolker B, Walker S. Fitting linear mixed-effects models using Ime4. J Stat Softw 20 I 5;67: I-48.

Brenner CA, Wolny YM, Barritt JA, Matt DW, Munne S, Cohen J. Mitochondrial DNA deletion in human oocytes and embryos. Mol Hum Reprod 1998;4:887-892.

Broekmans FJ, Soules MR, Fauser BC. Ovarian aging: mechanisms and clinical consequences. Endocr Rev 2009;30:465-493.

Chan CC, Liu WW, Lau EY, Yeung WS, Ng EH, Ho PC. Mitochondrial DNA content and 4977 bp deletion in unfertilized oocytes. Mol Hum Reprod 2005; I I:843-846.

Dalton CM, Szabadkai G, Carroll J. Measurement of ATP in single oocytes: impact of maturation and cumulus cells on levels and consumption. J Cell Physiol 20I4;229:353-36I.

de Carvalho BR, Rosa e Silva AC, Rosa e Silva JC, dos Reis RM, Ferriani RA, Silva de Sa MF. Ovarian reserve evaluation: state of the art. J Assist Reprod Genet 2008;25:31 I-322.

Fragouli E, Spath K, Alfarawati S, Kaper F, Craig A, Michel CE, Kokocinski F, Cohen J, Munne S, Wells D. Altered levels of mitochondrial DNA are associated with female age, aneuploidy, and provide an independent measure of embryonic implantation potential. PLoS Genet 20 I 5; I I:e I00524I.

Giles RE, Blanc H, Cann HM, Wallace DC. Maternal inheritance of human mitochondrial DNA. Proc Natl Acad Sci USA 1980;77:6715-6719.

Gut $P$, Verdin E. The nexus of chromatin regulation and intermediary metabolism. Nature 2013;502:489-498.

Hammond ER, Green MP, Shelling AN, Berg MC, Peek JC, Cree LM. Oocyte mitochondrial deletions and heteroplasmy in a bovine model of ageing and ovarian stimulation. Mol Hum Reprod 2016;22:26 I-27l .

Hauswirth W, Laipis P. Transmission genetics of mammalian mitochondria: a molecular model and experimental evidence. In: Quagliarello (ed) Achievements and Perspectives of Mitochondrial Research. Amsterdam: Elsevier Biomedical, 1985, 49-59.

Jacobs L, Gerards M, Chinnery P, Dumoulin J, de Coo I, Geraedts J, Smeets $\mathrm{H}$. mtDNA point mutations are present at various levels of heteroplasmy in human oocytes. Mol Hum Reprod 2007; I 3: I 49-I 54.

Jansen RP, de Boer K. The bottleneck: mitochondrial imperatives in oogenesis and ovarian follicular fate. Mol Cell Endocrinol 1998; 145:8I-88. 
Keefe DL, Niven-Fairchild T, Powell S, Buradagunta S. Mitochondrial deoxyribonucleic acid deletions in oocytes and reproductive aging in women. Fertil Steril 1995;64:577-583.

Krishnan KJ, Greaves LC, Reeve AK, Turnbull D. The ageing mitochondrial genome. Nucleic Acids Res 2007;35:7399-7405.

Lynch M, Koskella B, Schaack S. Mutation pressure and the evolution of organelle genomic architecture. Science 2006;3 I I:1727-1730.

May-Panloup P, Boucret L, Chao de la Barca JM, Desquiret-Dumas V, Ferre-L'Hotellier V, Moriniere C, Descamps P, Procaccio V, Reynier P. Ovarian ageing: the role of mitochondria in oocytes and follicles. Hum Reprod Update 2016;22:725-743.

Meldrum DR, Casper RF, Diez-Juan A, Simon C, Domar AD, Frydman R. Aging and the environment affect gamete and embryo potential: can we intervene? Fertil Steril 20 16; 1 05:548-559.

Mishra P, Chan DC. Mitochondrial dynamics and inheritance during cell division, development and disease. Nat Rev Mol Cell Biol 20 I 4; 1 5:634-646.

Otten $A B$, Smeets HJ. Evolutionary defined role of the mitochondrial DNA in fertility, disease and ageing. Hum Reprod Update 2015;21:67I-689.

Otten AB, Stassen AP, Adriaens M, Gerards M, Dohmen RG, Timmer AJ, Vanherle SJ, Kamps R, Boesten IB, Vanoevelen JM et al. Replication errors made during oogenesis lead to detectable de novo mtDNA mutations in zebrafish oocytes with a low mtDNA copy number. Genetics 2016;204:1423-1431.

Poe BG, Navratil M, Arriaga EA. Absolute quantitation of a heteroplasmic mitochondrial DNA deletion using a multiplex three-primer real-time PCR assay. Anal Biochem 2007;362: 193-200.

R Core Team. R: A Language and Environment for Statistical Computing. Vienna, Austria: R Foundation for Statistical Computing, 2016. https:// www.R-project.org/. URL.
Rebolledo-Jaramillo B, Su MS, Stoler N, McElhoe JA, Dickins B, Blankenberg D, Korneliussen TS, Chiaromonte F, Nielsen R, Holland $M M$ et al. Maternal age effect and severe germ-line bottleneck in the inheritance of human mitochondrial DNA. Proc Natl Acad Sci USA 2014; I I I:I5474-15479.

Ross JM, Stewart JB, Hagstrom E, Brene S, Mourier A, Coppotelli G, Freyer C, Lagouge M, Hoffer BJ, Olson L et al. Germline mitochondrial DNA mutations aggravate ageing and can impair brain development. Nature 2013;50 I:4I2-4I5.

Shaeib F, Khan SN, Ali I, Thakur M, Saed MG, Dai J, Awonuga AO, Banerjee J, Abu-Soud HM. The defensive role of cumulus cells against reactive oxygen species insult in metaphase II mouse oocytes. Reprod Sci 2016;23:498-507.

Stewart JB, Chinnery PF. The dynamics of mitochondrial DNA heteroplasmy: implications for human health and disease. Nat Rev Genet 2015;16: 530-542.

Stewart JB, Larsson NG. Keeping mtDNA in shape between generations. PLoS Genet 2014; 10:el004670.

te Velde ER, Pearson PL. The variability of female reproductive ageing. Hum Reprod Update 2002;8: I4I-I54.

Trifunovic A, Larsson NG. Mitochondrial dysfunction as a cause of ageing. J Intern Med 2008;263: I 67-I78.

Tsai HD, Hsieh YY, Hsieh JN, Chang CC, Yang CY, Yang JG, Cheng WL, Tsai FJ, Liu CS. Mitochondria DNA deletion and copy numbers of cumulus cells associated with in vitro fertilization outcomes. J Reprod Med 2010;55:491-497.

Wai T, Teoli D, Shoubridge EA. The mitochondrial DNA genetic bottleneck results from replication of a subpopulation of genomes. Nat Genet 2008;40: |484-1488. 